\title{
Structural Polypeptides of the Murine Coronavirus DVIM
}

\author{
By \\ K. Sugryama, R. Ishikawa, and N. Fukuhara \\ Department of Biology, Faculty of Science, \\ Hirosaki University, \\ Hirosaki, Japan \\ With 3 Figures \\ Accepted July 16, 1985
}

\begin{abstract}
Summary
The structural polypeptides of the murine coronavirus DVIM (diarrhoea virus of infant mice) have been analysed in comparison with other strains MHV-2, MHV-3, MHV-4(JHM) and MHV-S by SDS-PAGE. In the presence of 2-mercaptoethanol, three major glycopolypeptides, gp 180, gp 69, gp 25 (as a group of similar species) and one major non-glycosylated polypeptide $\mathrm{p} 58$ were detected. The gp 69 is a DVIM specifie glycopolypeptide, in which the glycosidic moieties are linked to the core polypeptide through $\mathrm{N}$ glycosidic bonds, and hence may be correlated with the short projections of the viral envelope. Further gp 140, which appears in the absence of reducing agents, is apparently a dimer of gp 69 held together by disulfide linkages. The gp 25 family, on the other hand, consists of four polypeptides, two of which are not metabolically inhibited by tunicamycin suggesting that they are O-linked glycopolypeptides. DVIM seems to be serologically closely related to the MHV-S strain as shown by neutralization.
\end{abstract}

\section{Introduetion}

Coronaviruses are a group of enveloped viruses which contain large single-stranded RNA genomes of messenger polarity $(12,14,17,22)$. However, the murine coronaviruses can be distinguished from the other coronaviruses by the presence of O-linked oligosaccharides $(4,9)$ Our previous studies on the morphology and some of the biological activities of the murine coronavirus strain DVIM (diarrhoea virus of infant mice) $(18,19)$ have revealed the presence of characteristic short projections located on the base of 'club-shaped coronaradiation' of the DVIM virion. We were 
therefore interested in studying the structural polypeptide of this virus especially in comparison with those of the well characterized murine coronaviruses.

In this report we describe the characteristic major glycopolypeptide gp 69 , which is exclusively detected on the DVIM and probably corresponds to the short projections. We also describe the effect of tunicamycin on the synthesis of the various structural polypeptides and the degree of immunocross reactivity between these DVIM proteins.

\section{Materials and Methods}

Virus and Cells

The mouse enteric coronavirus (DVIM) was adapted for growth in DBT cells using Eagle's MEM and supplemented with 7 per cent $(\%)$ calf serum and 3 per cent tryptose phosphate broth (DIFCO Lab.). The virus was cloned by three consecutive plaque passages. In addition the mouse hepatitis virus strains MHV-2, MHV-3, MHV-4 (JHM) and MHV-S, obtained from Dr. K. Fujiwara (University of Tokyo, Tokyo, Japan), as DBT cell culture suspensions, respectively were also cloned and purified by a similar procedure as described previously (18).

\section{Isotopic Labelling of Viral Polypeptides}

Viruses at MOI of 1.0 were allowed to adsorb to confluent monolayers of DBT cells for 90 minutes at $4^{\circ} \mathrm{C}$, following which cells were washed twice in Dulbeceo's PBS and fed with MEM that has been supplemented with 2 per cent of dialysed calf serum. Methionine concentration in MEM was reduced to one-tenth of that in normal medium. Four hours after infection, the medium was replaced with methionine-free MEM containing 2 per cent dialysed calf serum and $25 \mu \mathrm{Ci} / \mathrm{ml}$ of ${ }^{35} \mathrm{~S}$-methionine $\left(1200 \mathrm{Ci} / \mathrm{mmol}\right.$ : Amersham Corp.). DVIM was also labelled with ${ }^{14} \mathrm{C}$-glucosamine (56.8 mCi/mmol: Amersham Corp.), 2-8H-D-mannose (22 Ci/mmol : ICN Radio chemicals) and 5-, 6, ${ }^{3} \mathrm{H}-\mathrm{L}$-fucose (67 Ci/mmom: Amersham Corp.) at $5 \mu \mathrm{Ci} / \mathrm{ml}$ by the same procedure without a reduction of methionine, respectively. In some experiments, after virus adsorption tunicamycin (Sigma Chem. Co.) was added at a final concentration of $4 \mu \mathrm{g} / \mathrm{ml}$. In all experiments virions were harvested 10 hours after infection, and were purified as deseribed (18).

\section{Polyacrylamide Gel Electrophoresis (PAGE)}

Samples were analysed on 12 per cent slab gels by the method of LAEmura (6). The proteins were treated with sample buffer, boiled for 3 minutes and separated by slab gel at $12 \mathrm{~mA}$ for 18 hours. Gels were then prepared for fluorography (1) and exposed to Kodak X-Omat $R$ film at $-70^{\circ} \mathrm{C}$.

\section{Neutratization Test and Immunoprecipitation}

Hyperimmune sera against purified virions of each strain were prepared in rabbits as described previously (19). For virus neutralization, 100 TCID $_{50}$ of virus was incubated with fourfold dilutions of antiserum for 1 hour at $37^{\circ} \mathrm{C}$ in a final volume of $0.1 \mathrm{ml}$. The incubation mixture was then transferred to monolayers of DBT cells in microplates using four replicas per virus-serum dilution. The test was scored for cytopathology after 48 hours at $37^{\circ} \mathrm{C}$. For immunoprecipitation, purified ${ }^{35} \mathrm{~S}$-methionine labelled virions were disrupted with 2 per cent Nonidet-P 40 in NTE $(0.1 \mathrm{M} \mathrm{NaCl}, 0.01$ we Tris. 
Cl, 0.001 m EDTA, pH 7.3) buffer and $400 \mu$ of this disrupted viral components was then incubated with 15 ll of antiserum for 60 minutes at $37^{\circ} \mathrm{C}$ and then mixed with $200 \mu 1$ of 10 per cent formalin-fixed solution of S. aureus (MILES Sci.) dissolved in NTE buffer. After incubation for 60 minutes at $37^{\circ} \mathrm{C}$, the immunocomplexes bound to $S$. aureus were washed four times in NTE containing 0.1 per cent Triton-X by centrifugation and the final pellet was suspended in the sample buffer, containing 2 mercaptoethanol and boiled for 3 minutes. $S$. aureus was deposited by low speed centrifugation and the supernatants were then loaded on to the gels.

\section{Results}

\section{Cross-Neutralizing Antibodies Induced by Cloned Murine Coronaviruses}

The immunological relationship between DVIM and other well characterized strains of murine coronaviruses was examined by cross-neutralization (Table 1). DVIM was found to be very closely related with the HMV-S strain. In addition, bilateral minor cross-reactions between DVIM and MHV-2, or MHV-3, were also observed. In contrast, DVIM showed only weak cross-reaction with MHV-4 (JHM). A strong unilateral cross-reaction was also detected between DVIM and the anti-MHV-2 serum. These results, therefore, showed that in general DVIM is serologically related to the other strains of murine coronaviruses, particularly to MHV-S.

Table 1. Serological relationships of murine coronaviruses

\begin{tabular}{|c|c|c|c|c|c|c|}
\hline \multirow[b]{3}{*}{ Test } & \multirow[b]{3}{*}{ Antigen } & \multicolumn{5}{|c|}{ Antibody titer } \\
\hline & & \multicolumn{5}{|c|}{ Antiserum } \\
\hline & & DVIM & MHV -2 & MHV -3 & MHV-4 & MHV-S \\
\hline \multirow[t]{5}{*}{ Neutralization } & DVIM & $>4096$ & 512 & 64 & 4 & 512 \\
\hline & MHV-2 & 32 & 1024 & 256 & 4 & 512 \\
\hline & MHV -3 & 32 & 512 & 1024 & 8 & 2048 \\
\hline & MHV -4 & 4 & 16 & 64 & 512 & 256 \\
\hline & MHV-S & 1024 & 256 & 128 & 4 & $>4096$ \\
\hline HAI & DVIM & 512 & $<8$ & $<8$ & $<8$ & $<8$ \\
\hline
\end{tabular}

Neutralizing antibody titers are expressed as the reciprocal of the serum, dilution inhibiting 50 per cent of the input virus infectivity $\left(100\right.$ TCID $\left._{50}\right)$. HAI titre are given for DVIM only HA alone DVIM haernagglutinates. Kaolin treated mouse red blood cells were used

\section{Structural Polypeptides of DVIM}

Fig. 1 a showed the SDS-PAGE profile of ${ }^{35}$ S-methionine labelled DVIM under reducing conditions, when compared with other murine coronaviruses. The approximate molecular weights of the virion polypeptides were determined by comparing their electrophoretic mobilities with those of com- 
mercially available standards (BIO-RAD) in 7.5 per cent polyacrylamide tube gels, according to the procedure of MAIZEL (8). DVIM has four major polypeptides, with molecular weights of $180,000,69,000,58,000$ and 25,000 , respectively. One minor polypeptide with a molecular weight of 90,000 was detected in some preparations. The smallest of the major polypeptide species produced a broad band or a group of three to four closely migrating bands. The PAGE profiles of DVIM were generally similar to those observed for other murine coronaviruses such as MHV-2, MHV-3, and MHV-4, except that one major band with a mol. wt. of 69,000 was detected in DIVM virions exclusively. MHV-S strain also had a polypeptide with a mol. wt. of 65,000 similar to the 69,000 of DVIM.

${ }^{14} \mathrm{C}$-glueosamine labelled DVIM was used to identify glycosylated peptides. Analyses revealed that the three major poly peptides with mol. wts. of 180,000 , 69,000 and 25,000 were all glycosylated, and hence were designated gp 180, gp 69 and gp 25 respectively, whereas the other major polypeptide with a mol. wt. of 58,000 , which was not glycosylated, was designated p 58 (Fig. 1 b). It is interesting that gp 180 was labelled with both, ${ }^{3} \mathrm{H}$-mannose and ${ }^{3} \mathrm{H}$ fucose, whereas the gp 25 family of polypeptides were labelled with only ${ }^{3} \mathrm{H}$-fucose. In addition, DVIM specifie gp 69 was labelled with ${ }^{3} \mathrm{H}$-mannose and not with ${ }^{3} \mathrm{H}$-fucose. These findings, therefore, indicate that the various DVIM glycopolypeptides differ from each other according to the composition of their sugar residue moieties.

The presence of disulfide bonds in the structural polypeptides was studied by SDS-PAGE analysis of ${ }^{35}$ S-methionine labelled DVIM in the absence of 2-mercaptoethanol when compared with those of MHV-S. Under these conditions, gp 69 of DVIM could no longer be detected and instead a new species of polypeptide with a mol. wt. of 140,000 was observed (Fig. 1c). This suggested that this polypeptide is a disulfide-linked dimer of two smaller glycopolypeptides gp 69. MHV-S also had a similar disulfide-linked oligomeric species.

\section{Immunocross-Reactivity of DVIM Polypeptide}

In order to study the serological cross-reactivity between the DVIM polypeptides and those of the other MHV strains, ${ }^{35} \mathrm{~S}-$ methionine labelled

Fig. 1. Electrophoretic analysis of DVIM polypeptides. a PAGE profile of DVIM. ${ }^{35} \mathrm{~S}$-methionine labelled DVIM was electrophoresed in 12 per cent gel under reducing conditions. ${ }^{35} \mathrm{~S}$-labelled MHV type 2, 3, 4, and $\mathrm{S}$ were also shown in comparison with DVIM. $b$ Incorporation of sugar residues to DVIM polypeptides. ${ }^{14} \mathrm{C}$-glucosamine $(G)$, ${ }^{3} \mathrm{H}$-fucose $(F)$ and ${ }^{3} \mathrm{H}$-mannose $(M)$ labelled DVIM were electrophoresed as shown in (a). $v$ virion control. $c$ Effects of 2 -mercaptoethanol in DVIM polypeptides. ${ }^{35} \mathrm{~S}$. methionine labelled DVIM $(D)$ were electrophoresed in the absence $(-$ ) or presence $(+)$ of 2-ME. MHV-S $(S)$ were also shown in the same conditions respectively. The arrow indicates the new polypeptide of DVIM 

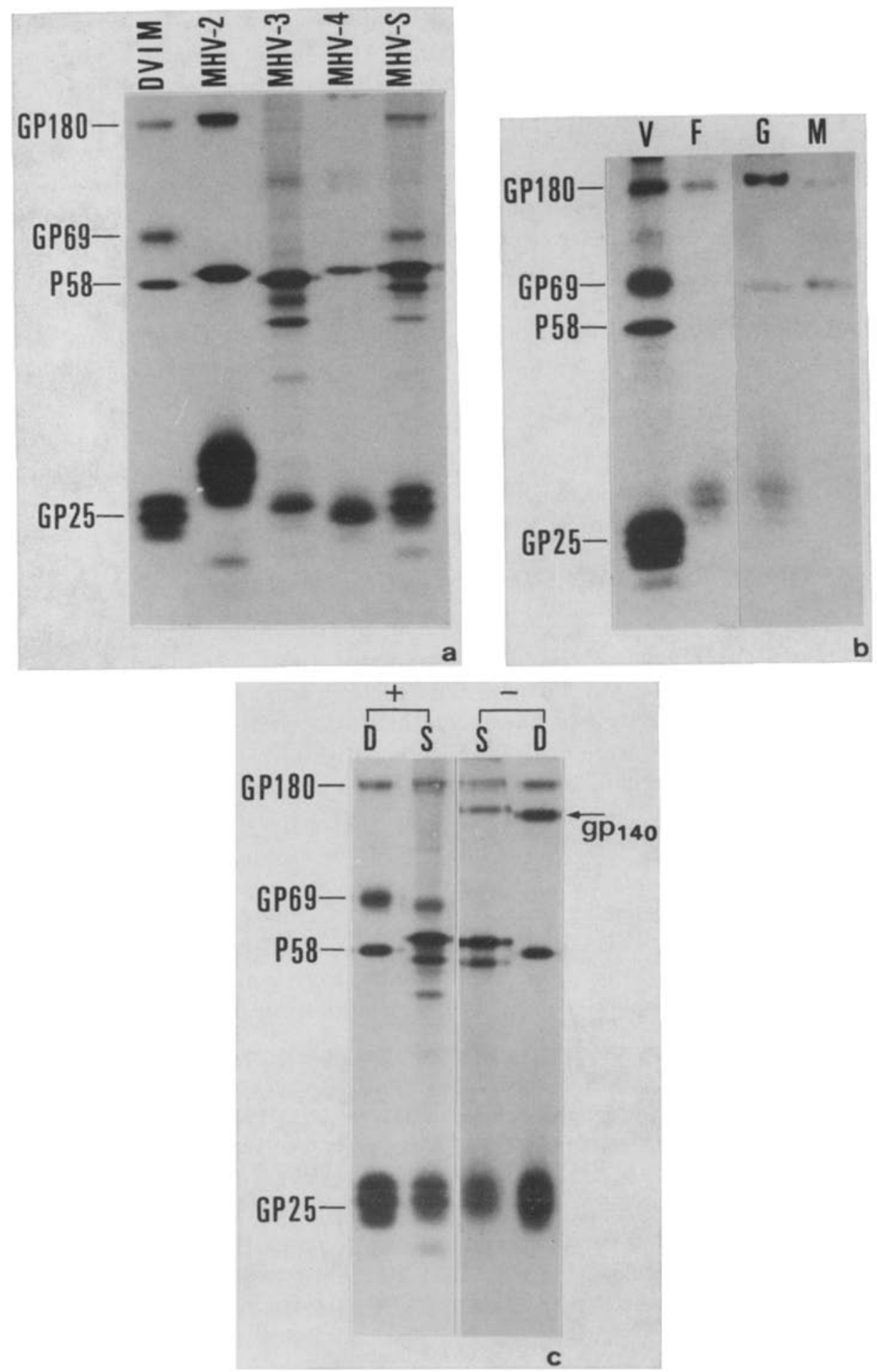
DVIM virions were immunoprecipitated employing antisera specific against individual strains, and assayed by SDS-PAGE under reducing conditions (Fig. 2a). Thus using homologous antisera all the structural polypeptides of DVIM were precipitated. The minor bands, which migrated slightly faster than the p58 species, as detected by immunoprecipitation, were assumed to be degradation products, most probably of the nucleocapsid protein. On the other hand, immunoreactions with heterologous antisera indicated that whereas there was strong cross-reactivity with p 58 of DVIM, there were only faint cross-reactions with both gp 180 and gp 25, respectively. It seems, however, that gp 180 shows considerable cross-reaction with the anti-MHV-S serum. In contrast, the DVIM specific polypeptide gp 69 was not immunologically related to any of the glycoproteins of the other murine coronaviruses.

As shown in Fig. 2b almost all of the strain-specific polypeptides immunoreacted with the anti-DVIM serum in varying degrees indicating that all the structural proteins of DVIM, except $\mathrm{gp} 69$, are immunologically related to those of the other MHV strains.

\section{Effects of Tunicamycin on the Synthesis of DVIM Polypeptides}

To identify the type of glycosidic bonds present in the structural glycoproteins, DVIM infected DBT cells were treated with tunicamycin $(4 \mu \mathrm{g} / \mathrm{ml})$ as described earlier. Viral particles isolated from tunicamycin treated cells lacked both, infectivity and haemagglutinating activity (data not shown). These viral particles were analysed together with viruses from non-treated cells on SDS-polyacrylamide gels under reducing conditions (Fig. 3).

Results indicate that both gp 180 and the DVIM-specific glycopolypeptide gp 69 could no longer be detected following tunicamycin treatment of cells. In addition, it appears that gp 25 consists of four polypeptides, W, X, Y and $Z$. Of these the small glycopolypeptide $Y$ also disappeared completely. In contrast the synthesis of both $W$ and $X$ was not affected and $Z$ was only partially affected by this drug. These results, together with the ability of mannose to be incorporated into the polypeptide (Fig. $1 \mathrm{~b}$ ), indicated that

Fig. 2. Immunoprecipitation of structural polypeptides. a Immunoprecipitation reactions of DVIM polypeptides with anti-MHV or anti-DVIM serum. Solubilized polypeptides of ${ }^{35} \mathrm{~S}$-methionine labelled DVIM were immunoreacted with antiserum prepared to MHV-2 (2), MHV-3 (3), MHV-4 (4), MHV-S (S) and DVIM (D) respectively. Immunocomplexes were dissolved in the presence of $2-\mathrm{ME}$, and electrophoresed. Coelectrophoresed DVIM was also shown (V). $O$ origin of the run. $b$ Immunoprecipitation reactions of MHVs polypeptides, with anti-DVIM serum. Solubilized polypeptides of ${ }^{35}$ S-methionine labelled MHV-2 (2), MHV-3 (3), MHV-4 (4) and MHV-S (S) were immunoreacted with anti-DVIM respectively, and electrophoresed as (a). Homologous reaction of DVIM was also shown in (D). $O$ origin of the run. 
the carbohydrate side chains of gp 180 and gp 69 are linked to the individual polypeptides through $\mathrm{N}$-glycosidic bonds $(9,20,21)$.
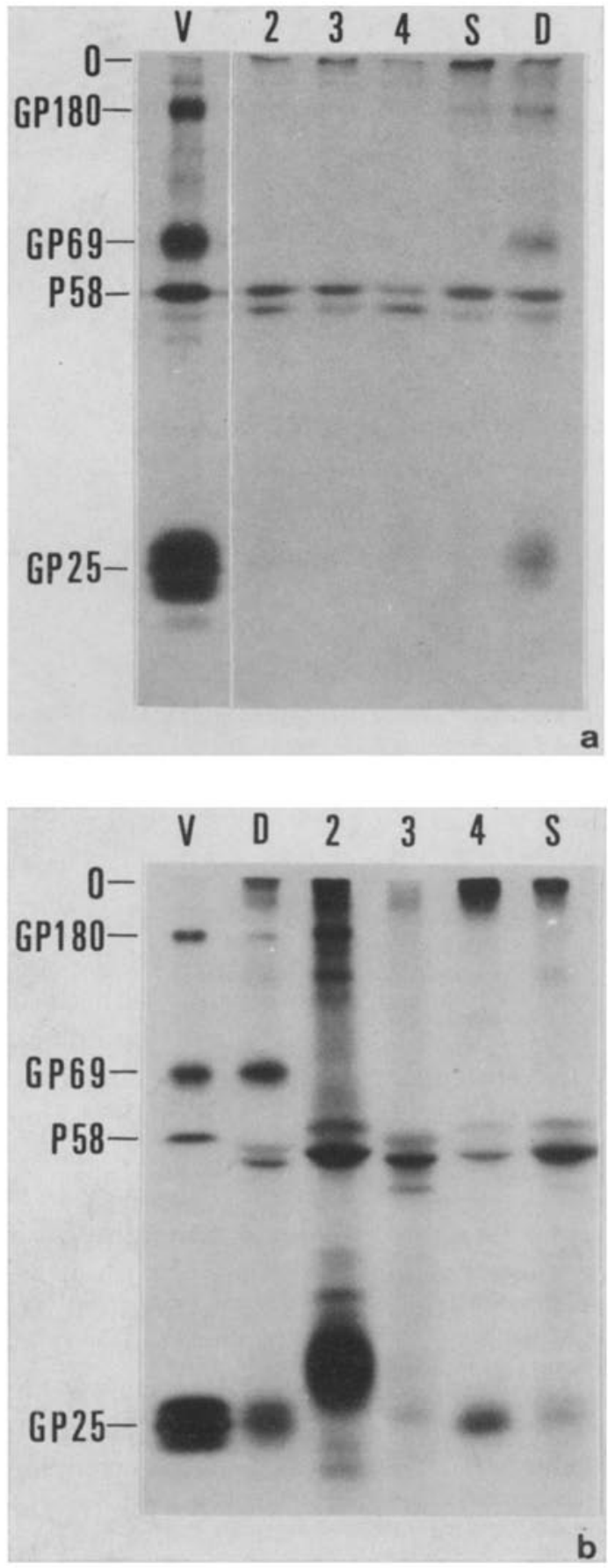


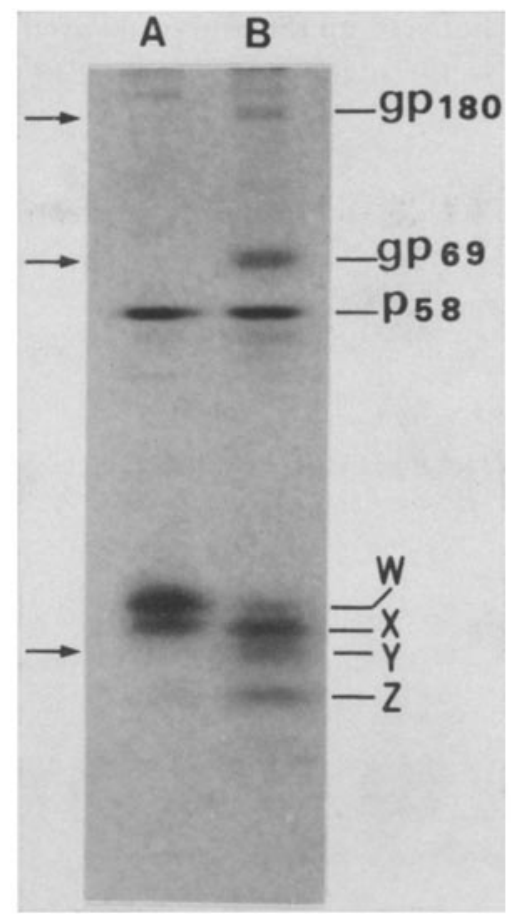

Fig. 3. Effects of tunicamycin on DVIM virion synthesis. ${ }^{35}$ S-methionine labelled DVIM virions, isolated from tunicamycin treated cells, were dissolved in the presence of 2-ME and analyzed in 12 per cent gel (A). Missing bands are shown by arrows. (B) virion control

\section{Diseussion}

The glycopeptide gp 180 appears to be equivalent to the largest glycoprotein, present in the well-characterized mouse hepatitis viruses, and we think it is a peplomeric protein in accordance with the findings of other investigators $(7,14,15,16,17,23)$. Although the minor glycopolypeptide gp 90 was weakly detectable in gels, it is also assumed to be a peplomeric protein and hence is probably associated with gp 180 by noncovalent bonds (17).

Gp69, on the other hand, was detected exclusively on the DVIM virions as a major structural polypeptide. Removal of the large projections of DVIM, by proteolytic digestions, resulted in the loss of both gp 180 and gp 90 from the intact DVLM virions. However, the viral particles still retained their haemagglutinating activity and these correlated with the presence of gp 69 (data not shown). Therefore, this suggested a close relationship between the small granular projections and the viral haemagglutinating activity. We therefore speculate that gp 69 is a structural component of the small granular projections which are located close to the envelope. We presume (Fig. 1c) that gp 69 is a subunit of the larger glycoprotein gp 140, which is 
bound by disulfide linkages in an oligomeric structure. Interestingly, a similar disulfide linked glycopolypeptide has been described for the porcine haemagglutinating encephalomyelitis virus and for the bovine coronavirus, and it was suggested that this polypeptide may represent haemagglutinin $(2,5,10,11)$. Generally, therefore, the haemagglutinating coronaviruses may share these oligomeric proteins as a distinctive structural feature in the form of small granular projections. Our studies also revealed that p58 was unaffected by protease treatment (data not shown) and hence were apparently protected by the viral envelope. This suggested that p58 was located internally and hence can be considered as a nucleoprotein as has been proposed by other workers $(2,3,7,14,15,23)$.

In this study we found that the gp 25 family of proteins were only slightly affected by protease treatment hence we are inclined to assume that they are embedded within the viral envelope. As was shown in Fig. 3, this family of glycoproteins consisted of four polypeptide species, designated $W, X, Y$ any $Z$. In gels these polypeptides gave bands that corresponded with those reported by RotTIER et al. (13) and were comparable to the bands of gp 26.5/E 1, gp 25.5/E1, gp 24.5/E 1 and $\mathrm{p} 22 / \mathrm{X}$ of $\mathrm{MHV}-\mathrm{A} 59$, respectively. Further, the synthesis of both $W$ and $X$ was not affected by tunicamycin treatment, suggesting that the carbohydrate side chains of these proteins may be linked to polypeptides through an $O$-glycosidic bonds $(4,9)$. In fact, the evidence for the existence of this type of bonds in DVIM was indicated by their sensitivity to alkali treatment under $\beta$-elimination conditions (data not shown). In contrast the synthesis of the $Y$ protein as well as gp 180 and gp 69 was completely inhibited by tunicamycin treatment. However, we failed to detect any ${ }^{3} \mathrm{H}$-mannose following labelling, which is characteristic for the $\mathrm{N}$-glycosidic bond glycoprotein (9), in the gp 25 family. Therefore, at present we cannot define the type of glycosidic bonds in the $\mathrm{Y}$ protein.

\section{Acknowledgment}

This work was supported in part by The Naito Foundation Grant No. 82-118.

\section{References}

1. Bonner, W. M., LAskey, R. A.: Film detection method for tritium-labeled proteins and nucleic acid in polyacrylamide gels. Eur. J. Biochem. 46, 83-88 (1974).

2. Callebaut, P. E., Pensaert, M. B.: Characterization and isolation of structural polypeptides in haemagglutinating encephalomyelitis virus. J. gen. Virol. 48, $193-204(1980)$.

3. Hrerholzer, J. C., Palmer, E. L., Whitfteld, S. G., Kaye, H. S., Dowdle, W. R.: Protein composition of coronavirus OC 43. Virology 48, 516-527 (1972).

4. Holmes, K. V., Doller, E. W., STuRMAN, L. S.: Tunicamycin resistant glyeosylation of coronavirus glycoprotein - Demonstration of a novel type of viral glycoprotein. Virology 115, $334-344$ (1981). 
5. KING, B., Brtan, D. A.: Bovine coronavirus structural proteins. J. Virol. 42, $700-707$ (1982).

6. Lafmmur, U. K.: Cleavage of structural proteins during the assembly of the head of bacteriophage T 4 . Nature (London) 227, 680-685 (1970).

7. Macnatghton, M. R.: The polypeptides of human and mouse coronaviruses. Arch. Virol. 63, $75-80(1980)$.

8. MAtzet, J. V.: Acrylamide-gel electrophorograms by mechanical fractionation; Radioactive adenovirus protein. Science 151, 988-990 (1966).

9. Nwmann, H., KLENK, H.-D.: Coronavirus glycoprotein E1, a new-type of viral glycoprotein. J. Mol. Biol. 153, 993-1010 (1981).

10. Pocock, D. H.: Effect of sulphydryl reagents on the biological activities, polypeptide composition, and morphology of haemagglutinating encephalomyelitis virus. J. gen. Virol. 40, 93-101 (1978).

11. Pocock, D. H., Garwes, D. J. : The polypeptides of haemagglutinating encephalomyelitis virus and isolated subviral particles. J. gen. Virol. 37, 487-499 (1977).

12. Roвb, J. A., Bond, C. W.: Coronaviridae. Compr. Virol. 14, 193-247 (1979).

13. Rottier, P. J. M., Horzinek, M. C., va. der ZeiJst, B. A. M.: Viral protein synthesis in mouse hepatitis vimus strain A 59 infected cells: effect of tunicamycin. J. Virol. 40, $350 \quad 357$ (1981).

14. Siddeld, S., Wege, H., TER Meulen, V.: The structure and replication of coronaviruses. Curr. Top. Microbiol. Immunol. 99, 131-163 (1982).

15. Sturkan, L. S.: Characterization of coronavirus. I. Structural proteins: Effects of preparative conditions on the migration of protein in polyacrylamide gels. Virology 77, 637-649 (1977).

16. Sturman, L. S., Hotmes, K. V.: Characterization of a coronavirus. II. Glyeoproteins of the viral envelope: Tryptic peptide analysis. Virology 77, 650-660 (1977).

17. Sturman, L. S., Holmes, K. V.: The molecular biology of coronaviruses. Adv-Virus Res. 28, 35-112 (1983).

18. Sugryama, K., Amano, Y.: Hemagglutination and structural polypeptides of a new coronavirus associated with diarrhea in infant mice. Arch. Virol, 66, 95-105 (1980).

19. Sugtyama, K., Amano, Y.: Morphology and biological properties of a new coronavirus associated with diarrhea in infant mice. Areh. Virol. 67, 241-251 (1981).

20. Takatsuki, A., Kohno, K., Tamura, G.: Inhibition of biosynthesis of polyisoprenol sugars in chick embryo microsomes by tunicamycin. Agric. Biol. Chem. 39, 2089-2091 (1975).

21. TKacz, J. S., LaMPEN, J. O.: Tunicamycin inhibition of polyisoprenyl N-acetylglucosaminyl pyrophosphate formation in calf liver microsomes. Biochem. Biophys. Res. Commun. 65, 248-257 (1975).

22. Tyrrelt, D. A. J., Almeida, J. D., Cunnmegam, C. H., Dowdle, W. R., Hohstad, M. S., MacIntosh, K., Tajima, M., Zakstelskaya, L. A., Easterday, B. C., KaptKian, A., Bingham, R. W.: Coronaviridae. Intervirology 5, 76-82 (1975).

23. Wege, H., Whge, H., Nagashima, K., Ter Meulen, V.: Structural polypeptides of the murine coronavirus JHM. J. gen. Virol. 42, 37-47 (1979).

Authors' address: Dr. K. Sugiyama, Department of Biology, Faculty of Science, Hirosaki University, Hirosaki, 036, Japan. 